\title{
PRINCIPIOS ÉTICOS Y JURÍDICOS DEL DERECHO GENÉTICO EN LAS DECLARACIONES INTERNACIONALES RELACIONADAS CON LAS INTERVENCIONES SOBRE EL GENOMA HUMANO*
}

\author{
ETHICAL AND JURIDICAL PRINCIPLES \\ OF GENETIC LAW IN INTERNATIONAL \\ DECLARATIONS RELATED TO \\ INTERVENTIONS ON HUMAN GENOMA
}

Ana Isabel Gómez Córdoba**

Fecha de recepción: 13 de enero de 2010 Fecha de aceptación: 22 de febrero de 2010

* Este artículo hace parte de los trabajos desarrollados en el marco del doctorado en Ciencias Jurídicas de la Pontificia Universidad Javeriana.

** Médico pediatra especialista en gerencia en salud pública y en derecho médico sanitario de la Universidad del Rosario, magíster en Bioética de la Universidad del Bosque, actualmente realiza estudios de doctorado en Ciencias Jurídicas en la Universidad Javeriana. Se desempeña como Director del Programa de Medicina y como codirector de la especialización de Derecho Médico Sanitario de la Universidad del Rosario. Pertenece al grupo de investigación en educación, línea de investigación en bioética y derecho médico sanitario, de la Universidad del Rosario reconocido por Colciencias. Bogotá, Colombia.

Correo electrónico: anai.gomez@urosario.edu.co 


\section{RESUMEN}

El desarrollo del conocimiento genómico aplicado a la salud humana ofrece grandes beneficios bajo la forma de medicina genómica, predictiva o reparativa; sin embargo, conlleva profundas implicaciones de índole social, política, ética, económica e ideológica, las cuales requieren un marco regulatorio, que de manera genérica se denomina derecho genético. Este artículo pretende caracterizar el derecho genético únicamente en lo referente a las intervenciones en el genoma humano en el área de la biomedicina, desde los principios éticos y jurídicos que deben hacer parte de su fundamentación filosófica, para tal fin se analizarán las más importantes declaraciones emanadas por los organismos y las asociaciones de carácter internacional relacionadas con el tema, para identificar los principios éticos y jurídicos que deben orientar la caracterización del derecho genético.

Palabras clave: genoma humano, bioderecho, derecho genético, principios éticos, principios jurídicos, declaraciones internacionales.

Palabras clave: Genoma humano, legislación, ética médica, medicina legal.

\section{ABSTRACT}

The development of genomic knowledge applied to human health offers great benefits in the form of genomic, predictive or repair medicine; nevertheless carries profound implications for social, political, ethical, economic and ideological, which require an effective regulatory framework, which is known generically genetic law. This article aims to characterize the genetic law only with regard to interventions in the human genome in the area of biomedicine, from the ethical and legal principles that must be part of its philosophical foundation to this en end we analyze the most important statements issued agencies and international associations related to the subject to identify the ethical and legal principles that should guide the characterization of the genetic right.

Key words: human genome, bio law, genetic law, ethical principles, legal principles, international declarations.

Key words plus: Genome, Human, Legislation, Medical ethics, Forensic Medicine. 


\section{INTRODUCCIÓN}

La decodificación del genoma humano es sin lugar a dudas uno de los más importantes hitos científicos de la humanidad; la posesión de esta forma particular de conocimiento posibilita el desarrollo de innumerables productos y servicios que ofrecen beneficios para las personas en diversos ámbitos, especialmente en el de la salud bajo la forma de medicina genómica, predictiva, personalizada, de reemplazo y antienvejecimiento, los cuales son producto de los cuatro hitos que se reconocen en la historia de la genética reciente: el descubrimiento de la estructura de doble hélice, las técnicas de ingeniería genética, la clonación de la oveja Dolly y la decodificación del genoma humano, gracias al Proyecto genoma humano. Cuando la investigación en genética humana, es ética y pertinente, permite entre otras cosas: determinar, en cualquier momento de la vida (preconcepcional, prenatal o posconcepcional) o de la historia natural de la enfermedad, la probabilidad o propensión de padecer una alteración que dependa de una expresión génica, e intervenirla (mediante consejería genética o el uso de técnicas de ingeniería genética); diseñar medicamentos personalizados haciéndolos más eficaces y disminuyendo los efectos indeseables (fármacogenómica); clonar células madre que in vivo reparen tejidos dañados, para tratar padecimientos como el Alzhéimer (clonación terapéutica); desarrollar órganos para su reemplazo; intervenir en los mecanismos teloméricos para modular los procesos de envejecimiento; desarrollar nuevos agentes terapéuticos (como la insulina, el factor VII); o lograr un efecto terapéutico mediante la introducción de material genético en una célula o tejido para procurar determinada expresión génica en el individuo o sus futuras generaciones (terapia génica de células somáticas y germinales) $^{1}$; y la realización de pruebas genéticas en casos de paternidad, o en la comprobación de delitos. Sin embargo, el poder transformador de la intervención tecnológica sobre el genoma humano tiene profundas implicaciones de diversa índole, las cuales han sido objeto de profundos análisis por diversas disciplinas (como la bioética) que expresado en declaraciones internacionales establecen los lineamientos para el desarrollo de marcos regulatorios eficaces. Es así como la interacción de la vida con la tecnología requiere un progreso jurídico paralelo que garantice el mejor uso del conocimiento genómico, el acceso justo a los beneficios generados y la modulación de los posibles riesgos, en especial de aquellos de carácter grave e irreversible, que podrían afectar derechos individuales y colectivos y colocar en peligro la vida en el planeta ${ }^{2}$. Algunos de los riesgos que se asocian con las interven-

1 María Victoria Vidal Casero. "El proyecto genoma humano, sus ventajas, sus inconvenientes y sus problemas éticos". Cuadernos de bioética. Págs. 393-414. Vol. 12. n 46. (2001). (Ejemplar dedicado a: ¿En qué fundamentamos la bioética?).

2 Gonzalo Figueroa Yánez. Hacia una integración supranacional de los principios rectores sobre el 
ciones en el genoma humano son de manera general: el patentamiento de la vida y el acceso inequitativo a los beneficios, la discriminación genética, el reduccionismo y el determinismo genéticos, el desarrollo de quimeras e híbridos, los riesgos asociados con la bioseguridad y la instrumentalización del hombre, que incluye, el perfeccionamiento humano, la clonación reproductiva, la clonación terapéutica (cuando se trata de células madre provenientes de embriones humanos) y la investigación en seres humanos por fuera de pautas éticas y jurídicas de carácter internacional o nacional.

La cercana relación entre los temas jurídicos, biológicos y médicos, así como los conflictos originados en la interacción de la vida con la tecnología en el ámbito de la salud han dado origen al desarrollo de una nueva área del derecho, que en su conjunto ha recibido diferentes nombres: bioderecho, biolegislación, biojurídica, biojurisprudencia, derecho biológico, biotecnología jurídica, bionómica, derecho médico, derecho sanitario, derecho médico sanitario, derecho de la salud, derecho genético, entre otros. Con respecto a cuál denominación corresponde al género o a la especie, para algunos autores depende de su origen geográfico o del contexto en que sea empleado: el término bioderecho es más cercano a las denominaciones anglosajonas como bio law o bioethics law y el de biojurídica cercano a la tradición italiana. El término biojurídica se usa más en el contexto de la filosofía del derecho, y el de biolegislación, asociados a otras especialidades del derecho ${ }^{3}$, aunque otros autores consideran que la denominación derecho médico sanitario es más amplia e incluyente.

Para los fines de este artículo emplearé la denominación genérica de bioderecho, del cual se expondrán a continuación algunas definiciones: "Es el conjunto de disposiciones jurídicas, decisiones jurisprudenciales y principios del derecho aplicables a las acciones de investigación, de desarrollo tecnológico y de naturaleza clínica que incidan en la salud e integridad física de las personas, para que se ejerzan con responsabilidad, preservando la dignidad, la autonomía informativa, la seguridad jurídica y la integridad psicológica de los individuos, y la equidad social"4. En este contexto el derecho genético es "una rama del derecho que regula el desarrollo de la ciencia genética y su influencia en el ser humano", se encarga de "estudiar y normar todas aquellas actividades técnicas o científicas relacionadas con la composición génica del

genoma humano. Una visión personal desde la perspectiva latinoamericana. Págs. 231 a 245 . Acta Bioethica. Año VI. no 2. (2000).

3 Ángela Aparisi Miralles. Bioética, bioderecho y biojurídica, reflexiones desde la filosofía del derecho. Anuario de Filosofía del Derecho, ISSN 0518-0872, n 24. (2007). Págs. 63-84.

4 D. Valadez. Problemas del bioderecho y del derecho genómico. Panorama sobre la legislación en materia de genoma humano en América Latina y el Caribe. Pág 389. (2006). http://www.bibliojuridica. org/libros/5/2265/15.pdf 
hombre", brinda "una protección y seguridad jurídica al ser humano y a las relaciones sociales que se derivan de aquellos avances de la ciencia genética"s.

Ya en lo referente a la relación entre el derecho y el genoma humano, es importante resaltar que el derecho internacional relacionado con el genoma humano se expresa prioritariamente a través del derecho declaratorio o soft law o derecho de referencia, el cual es denominado por algunos como el "derecho universal de la bioética".

El presente artículo hace parte del desarrollo de mi tesis doctoral, la cual pretende caracterizar el derecho genético en Colombia referido a las intervenciones en el genoma humano, para tal fin se analizarán las más importantes declaraciones emanadas por organismos y asociaciones de carácter internacional relacionadas con el tema, para identificar los principios éticos y jurídicos que deben orientar la caracterización del derecho genético, denominado genéricamente de tipo soft law.

\section{PRINCIPIOS ÉTICOS Y JURÍDICOS INTERNACIONALES APLICABLES A LAS INTERVENCIONES EN EL GENOMA HUMANO}

Diversos organismos de carácter internacional (global, regional o disciplinar) han advertido los riesgos que conlleva la intervención sobre el genoma humano y han propuesto una serie de ideas (principios) fundamentales, que orienten la resolución de conflictos éticos y jurídicos relacionados con la intervención tecnológica sobre el genoma humano, expresadas en declaraciones y convenios. Cuando se trata de conflictos originados en la interacción de la vida con la tecnología, adquiere especial importancia para el derecho en su fundamentación la valoración axiológica, en el cual el debate ético precede al desarrollo jurídico?.

La Real Academia de la Lengua Española define principio como "la norma o idea fundamental que rige el pensamiento o la conducta". Los principios referidos a lo moral son aquellos que permiten que los actos sean buenos, que al orientar acciones, las consecuencias de éstas sean mejores que las que pudieran derivarse de cualquier otra acción alternativa. Los principios jurídicos son definidos como: "las ideas fundamentales sobre la organización jurídica de una comunidad, emanadas de la conciencia social, que cumplen funciones

5 Enrique VArsi. Fundamentos básicos del derecho genético. http://issuu.com/evarsi/docs/varsi-cap2

6 Noelle Lenoir. El genoma humano, la ética y los derechos humanos. Pág. 131. Diálogo México número 23. (1998)

7 Luisa Benavides de Castañeda. Bioética y bioderecho: temas para la reflexión. Pág. 91. Anuario no 30 (2007).

8 Roberto-Marino Jiménez Cano. Sobre los principios generales del derecho. Especial consideración. En Derecho Español. Revista Telemática de Filosofía del Derecho, nº 3. 1999/2000. Págs. 1-18. D.L. M-32727-1998. 
fundamentadora, interpretativa y supletoria con respecto a su total ordenamiento jurídico"; "los principios jurídicos son los pensamientos directores de una regulación jurídica existente o posible"; se entiende como principios ético-jurídicos las "pautas orientadoras de normación jurídica que, en virtud de su propia fuerza de convicción, pueden justificar decisiones jurídicas"10.

Los principios jurídicos deben diferenciarse de las reglas: "los principios configuran el caso de forma abierta, las reglas lo hacen de forma cerrada; mientras en las reglas las propiedades que conforman el caso constituyen un conjunto finito y cerrado, en los principios no puede formularse una lista cerrada de las mismas; mientras los principios prescriben que algo sea realizado en la mayor medida posible dentro de las posibilidades jurídicas y reales existentes, las reglas constituyen mandatos que prescriben (obligando, permitiendo o prohibiendo) una conducta determinada sin admitir otras excepciones que las expresamente previstas"11. Según DworkIN, existen dos tipos de principios: unos establecen metas o propósitos sociales, políticos o económicos mientras que otros establecen exigencias de justicia, equidad, libertad; unos hablan de lo correcto y bueno, y los otros de la utilidad.

A continuación se analizará el acervo jurídico del tipo soft law que se expresa en algunas de las más importantes declaraciones internacionales, de organizaciones internacionales o asociaciones disciplinares de carácter internacional, que busca identificar los principios éticos y jurídicos orientadores de la resolución de los problemas ético-jurídicos de la intervención sobre el genoma humano, anotando que provienen de fuentes diferentes y que tienen un carácter jerárquico distinto siendo de mayor relevancia las emitidas por organismos como la Unesco y la ONU. A continuación se presentan las fuentes seleccionadas para el análisis: ONU (Declaración Universal de los Derechos Humanos, 1948; Declaración sobre la clonación humana de las Naciones Unidas, 2005; el Convenio de las Naciones Unidas sobre la Diversidad Biológica del 5 de junio de 1992), Unesco (Declaración Universal sobre el Genoma Humano y los Derechos Humanos de 1997; la Declaración Internacional sobre los Datos Genéticos Humanos, 2003; la Declaración Universal de Bioética y Derechos Humanos del 2005 y, la Declaración Sobre la Ciencia y el Uso del Saber Científico, 1999, y la Declaración sobre las Responsabilidades de las Generaciones actuales para con las Generaciones Futuras, 1997; la Declaración Universal de la Unesco sobre la Diversidad Cultural del 2 de noviembre del 2001), Tribunal Internacional de Nüremberg:

9 JoAquín Arce y FlóRez-VAldés. Los principios generales del derecho y su formulación constitucional. Pág. 99. Madrid. Editorial Civitas. S.A.

10 Karl Larenz. Metodología de la ciencia del derecho. Barcelona. Editorial Ariel S.A. Traducción y revisión de Marcelino Rodríguez Molinero. (1994). (1ª ed. en Ariel. $3^{\mathrm{a}}$ ed. española. Pág. 418).

11 Carlos Ruiz Miguel. La nueva frontera del derecho a la intimidad: genética y dignidad. http://web. usc.es/ ruizmi/pdf/genet.pdf 
Código de Nüremberg, 1946, Asociación Médica Mundial (Declaración de la Asociación Médica Mundial sobre el Proyecto Genoma Humano, 1992; Declaración de Helsinki de la Asociación Médica Mundial sobre principios éticos para las investigaciones médicas en seres humanos, 2008), Asociación Mundial de Bioética (Declaración de la Sociedad Mundial de Bioética de Gijón, 2000).

Producto del análisis documental, se identificaron los siguientes principios: a) Dignidad humana (igualdad, individualidad, libertad), b) autonomía, c) beneficencia - no maleficencia, d) justicia, e) diversidad y pluralidad, f) cooperación y solidaridad, g) protección a generaciones futuras, h) libertad científica, i) protección a la propiedad intelectual, j) inclusión y k) precaución, los cuales se caracterizarán a continuación.

a) Dignidad humana (igualdad, no discriminación, libertad, individualidad): todas las declaraciones reconocen como premisa ética la dignidad humana, por la cual cada ser humano sin ningún tipo de distingo posea intrínsecamente el mismo valor y se encuentre en condiciones de igualdad en cuanto a su dignidad y derechos ${ }^{12}$. Dos principios del pensamiento kantiano se relacionan con este concepto: las personas no pueden ser utilizadas como medio, son fines por sí mismas, por lo tanto, se tiene la obligación positiva de tratar a las personas como fines (se puede tratar a las personas como medios siempre y cuando se traten también como fines); las personas tienen dignidad y no tienen precio. Por esto "la dignidad de la persona es la medida que debe guiar las intervenciones biológicas, genéticas y médicas sobre el hombre desde su concepción hasta su último suspiro"13. La dignidad humana, es consustancial al hecho de pertenecer a la especie humana, con la diversidad que esto implica ${ }^{14}$; se distinguen dos tipos de dignidad humana: la ontológica y la moral; la primera es común a todos los seres humanos, independiente de su capacidad de autodeterminación, racionalidad, autopercepción, comportamiento moral o sentido de trascendencia; acorde con esta dignidad todos somos iguales, merecedores del mismo respeto independientemente del género, la raza, la capacidad económica, la ideología, el momento del ciclo vital en que se encuentre o su material genético; la segunda, la dignidad moral, se relaciona con el comportamiento del sujeto, cuando este es acorde con lo que moralmente es considerado bueno ${ }^{15}$. Se reconoce además de la

12 Declaración Universal de Derechos Humanos. Organización de Naciones Unidas. Adoptada y proclamada por la Resolución de la Asamblea General 217 A (III) del 10 de diciembre de 1948: Preámbulo. Art. $1^{\circ}$.

13 Mercedes Alberruche Díaz-Flores. La clonación y selección de sexo ¿? Madrid. España. Ed. Dykinson. (1998).

14 Declaración Universal del Genoma Humano y los Derechos Humanos. Unesco. Aprobada el 11 de noviembre de 1997 por la Conferencia General en su $29^{a}$ reunión. Art. $1^{\circ}$.

15 Mariano Moreno Villa. Dignidad de las personas en el diccionario del pensamiento contemporáneo. Págs. 359-368. 
dimensión individual de la dignidad humana, una de carácter colectivo, la cual adquiere relevancia cuando se analiza el riesgo que implica para el patrimonio genómico de las futuras generaciones la intervención del material genético humano. Se hace evidente el riesgo que suponen para la dignidad humana las intervenciones biotecnológicas, por lo que el respeto a ella es principio rector del desarrollo científico y su aplicación en la biología y la medicina ${ }^{16,17}$.

La dignidad humana se encuentra relacionada con otros principios que deben orientar las investigaciones, estos son el respeto a la vida, a la libertad (que reconoce que el hombre es más que su condición genética, visión que supera el determinismo genético, evidenciando la naturaleza evolutiva del genoma a través de la mutación y su posibilidad de expresión individual dependiendo del entorno natural y social, su estado de salud, condiciones de vida, alimentación o educación) ${ }^{18}$, a la intimidad, a la identidad (derecho a la unicidad y reconocimiento de la identidad jurídica ${ }^{19}$, a la integridad (preservación de la unicidad y esencia genética, frecuentemente se asocia con el concepto de seguridad) ${ }^{20}$; a la no discriminación o estigmatización (de personas, familias, grupos o comunidades) en razón de la condición genética $^{21,22,23,24}$, la igualdad, la equidad y la justicia ${ }^{25}$ y a la diversidad (entendida en el contexto de interacción hombre-medio ambiente) ${ }^{26}$, los cuales serán objeto de análisis particular.

El respeto a la dignidad humana implica balancear la libertad científica con la autonomía y bienestar de las personas que participan en procesos de investigación ${ }^{27}$. El hombre no puede ser instrumentalizado en los procesos de investigación o de aplicación de técnicas de ingeniería genética, es una práctica que es contraria a la dignidad humana, con protección al principio de la autonomía o a la propia individualidad irrepetible (prohibición de clonación reproductiva, repetición de un ser humano idéntico a otro vivo o muerto, en el que coincide la totalidad de la serie de genes nucleares) o al uso del hombre como medio (prohibición de destrucción de embriones humanos

16 Unesco. Declaración sobre la ciencia y el uso del saber científico. Adoptada por la Conferencia mundial sobre la ciencia el $1^{\circ}$ de julio 1999. Art. 19.

17 Declaración de Helsinki. Asociación Médica Mundial. Adoptada en 1964, último ajuste Asamblea General de octubre 2008. Art. 11

18 Declaración Universal del Genoma Humano y los Derechos Humanos. Arts. $2^{\circ}$ (b), $3^{\circ}$.

19 Declaración de Derechos Humanos. Art. $5^{\circ}$.

20 Declaración de Derechos Humanos. Art. $3^{\circ}$.

21 Declaración de los Derechos Humanos. Art. $2^{\circ}$.

22 Declaración del Genoma Humano y los Derechos Humanos. Art. $6^{\circ}$.

23 Declaración de Bioética y Derechos Humanos. Aprobada por la Conferencia General de la Unesco octubre del 2005. Art. 11.

24 Declaración Internacional sobre los Datos Genéticos Humanos. Art. $7^{\circ}$.

25 Declaración de Bioética y Derechos Humanos. Art. 10.

26 Declaración de Bioética y Derechos Humanos. Arts. $2^{\circ}, 17$.

27 Declaración Bioética de Gijón. Sociedad Internacional de Bioética. Comité científico Congreso Mundial de Bioética (Gijón, España, 2000). 
para investigación o protección de éste cuando la legislación permita su uso), la alteración del material genético de futuras generaciones (intervención sobre líneas germinales) $)^{28,29,30}$.

También impide la comercialización, aprovechamiento, usufructo o patente del genoma humano (en su condición de patrimonio de la humanidad) en su estado natural o más pura expresión, así como de la información genética, proteómica y material biológico humano ${ }^{31,32}$. Con respecto al proceso de manejo (recolección, tratamiento, utilización y conservación) de los datos de información proteómica y material biológico humano la dignidad humana es principio rector ${ }^{33}$, asociado al hecho de que estos no sean motivos de discriminación o estigmatización, de individuos (relacionados con el comportamiento) y comunidades. Por esta razón se restringe el uso de pruebas predictivas en el área de la genética a aquellas que se realicen "con fines médicos o de investigación médica y con un asesoramiento genético apropiado" 34 .

b) Autonomía: los principios de autonomía, beneficencia, no maleficencia y justicia hacen parte de los principios fundamentales de la bioética enunciados por BEAUCHAMP y CHIDRESS ${ }^{35}$. El principio de autonomía reconoce el derecho que tiene cada individuo de tener su propio punto de vista, elegir y realizar acciones conforme a los valores y creencias personales, a la responsabilidad individual. Hace parte de la dignidad de todo ser humano, en lo relacionado con su libertad y racionalidad, bien sea que la persona pueda ejercerla por sí misma o deba ser protegido por otros debido a un estado especial de vulnerabilidad, es así que implica un autocontrol sobre las posiciones de poder ${ }^{36}$. La autonomía se relaciona estrechamente con el derecho al acceso a los propios datos genéticos, a la intimidad, identidad, especificidad y confidencialidad, específicamente en lo relacionado con el acceso a la información genética. La Declaración Universal sobre el Genoma Humano y los Derechos Humanos especifica: "sólo la legislación podrá limitar los principios de consentimiento y confidencialidad, de haber razones imperiosas para ello, y a reserva del estricto respeto del derecho internacional público y del derecho internacional relativo a los derechos humanos". Vale la pena mencionar que en la Decla-

28 Declaración sobre la Clonación Humana. Organización de Naciones Unidas, adoptada por la Asamblea General de las Naciones Unidas, el 23 de marzo del 2005.

29 Declaración Universal del Genoma Humano y los Derechos Humanos C. Art. 10.

30 Declaración de Bioética de Gijón.

31 Declaración Universal del Genoma Humano y los Derechos Humanos. Art. $4^{\circ}$.

32 Declaración de Bioética de Gijón.

33 Declaración Internacional sobre los Datos Genéticos Humanos. Unesco, aprobado por la Conferencia General en París, 16 de octubre del 2003. Art. $1^{\circ}$.

34 Declaración Internacional sobre Datos Genéticos. Art. $7^{\circ}$.

35 Tom Beauchamp \& James Chidress. Principles of Biomedical Ethics. Nueva York. Oxford University Press. (1979).

36 Declaración de Bioética y Derechos Humanos. Art. 5. 
ración de Bioética de Gijón se contextualiza la autonomía, en relación con los principios de justicia y solidaridad ${ }^{37,38,39,40,41}$.

La manera como se expresa la autonomía en las distintas declaraciones es a través del respeto a la libertad individual y, específicamente, en los procesos de investigación científica a través de la figura del consentimiento informado, el cual en lo relacionado con la genómica se entiende como: "el permiso específico, informado y expreso que una persona da libremente para que sus datos genéticos sean recolectados, tratados, utilizados y conservados ${ }^{42}$. Requiere de la competencia la persona que lo emite, libertad e información, expresados así:

La competencia se desarrolla progresivamente en el ser humano acorde con su neurodesarrollo, puede por tanto estar afectada por procesos patológicos (como la demencia) o por la edad (como en el caso de menores de edad). Las distintas declaraciones tienen en cuenta las situaciones en las cuales personas menores de edad o con limitaciones en su capacidad mental (estados especiales de vulnerabilidad) pueden participar en procesos de investigación, en cuyos casos se requerirá, entre otros requisitos, la voluntad de quien represente sus derechos ante la ley, la primacía del mejor interés, el beneficio directo de quien participa en la investigación y, en casos excepcionales, en aras de un interés superior ("sólo podrá efectuarse a título excepcional, con la mayor prudencia y procurando no exponer al interesado sino a un riesgo y una coerción mínimos, y si la investigación está encaminada a redundar en beneficio de la salud de otras personas pertenecientes al mismo grupo de edad o que se encuentren en las mismas condiciones genéticas, a reserva de que dicha investigación se efectúe en las condiciones previstas por la ley y sea compatible con la protección de los derechos humanos individuales) ${ }^{43}$. $\mathrm{Si}$ es posible, se debe buscar el asentimiento o participación del sujeto de investigación menor (que aumenta en proporción a la edad y al grado de madurez) o incompetente a causa de enfermedad, el no consentimiento debe ser respetado. De otra parte el consentimiento debe cumplir con los mismos

\footnotetext{
37 Declaración Internacional sobre Datos Genéticos Humanos. Art. 13.

38 Declaración Universal sobre el Genoma Humano y los Derechos Humanos. Arts. $7^{\circ}, 9^{\circ}$

39 Declaración de bioética y derechos humanos. Art. $9^{\circ}$.

40 Declaración de Helsinki. Art. 23.

41 Declaración de Bioética de Gijón.

42 Declaración Universal sobre el Genoma Humano y los Derechos Humanos B. Derechos de las personas interesadas. Art. $5^{\circ}$.

43 Declaración de Helsinki. Arts. 24, 34.
} 
elementos requeridos en los sujetos competentes, como son los de información, voluntad y revocatoria ${ }^{44,45,46,47}$.

En algunas de ellas se especifica que en los casos de no contar con la presencia del representante legal y que no pueda dilatarse más la investigación, esta podrá realizarse sin consentimiento informado "siempre que las razones específicas para incluir a individuos con una enfermedad que no les permite otorgar consentimiento informado hayan sido estipuladas en el protocolo de la investigación y el estudio haya sido aprobado por un comité de ética de investigación" ${ }^{\prime 4}$.

En los casos de investigación en genética humana se requiere el consentimiento para la recolección, tratamiento, uso, conservación y cruce de datos genéticos, proteómicos y material biológico humano (actual y almacenado), así como para cambios en las condiciones de uso, a no ser que se trate de un interés superior como el de la salud pública ${ }^{49}$.

La declaración de Helsinki establece que "podrá haber situaciones en las que será imposible o impracticable obtener el consentimiento para dicha investigación o podría ser una amenaza para su validez. En esta situación, la investigación sólo puede ser realizada después de ser considerada y aprobada por un comité de ética de investigación." 50,51

Libertad: la participación de las personas debe ser libre e inequívoca, esto implica la posibilidad de asentir o disentir. En todos los casos la voluntad esté exenta de vicios (error, fuerza y dolo) ${ }^{52}$. En el caso de investigaciones con fines médicos y científicos en las que se recolecten datos genéticos, datos proteómicos o muestras biológicas, las personas pueden revocar el consentimiento sin que acarree perjuicios, siempre y cuando sea técnicamente factible (esto no sería posible, por ejemplo, cuando los datos están irreversiblemente disociados de la persona), adicionalmente cuando no es posible determinar los deseos estos deben ser irreversiblemente disociados o destruidos ${ }^{53,54}$.

Información: no existe libertad sin alternativas y a éstas se accede a través de la información, la cual debe ser, entre otras características, previa, clara, objetiva, suficiente y apropiada al tipo de decisiones racionales que se deben tomar (generalmente asociada con los fines u objetivos, los beneficios calcu-

44 Declaración Universal sobre el Genoma Humano y los Derechos Humanos B. Derechos de las Personas Interesadas. Art. $5^{\circ}$.

45 Declaración de Bioética y Derechos Humanos. Art. $7^{\circ}$

46 Declaración Internacional sobre Datos Genéticos Humanos. Art. $8^{\circ}$.

47 Declaración de Helsinki. Arts. 27, 28, 29.

48 Declaración de Helsinki 29.

49 Declaración Internacional sobre los Datos Genéticos Humanos. Art. $2^{\circ}$.

50 Declaración de Helsinki: Art. 25.

51 Declaración Internacional sobre los Datos Genéticos Humanos. Art. 22.

52 Declaración de Helsinki. Arts. 22, 26.

53 Declaración Internacional sobre Datos Genéticos Humanos. Arts. $8^{\circ}, 9^{\circ}$.

54 Código de Nüremberg. Tribunal Internacional de Nüremberg. 1947. Art. $9^{\circ}$ 
lados y riesgos previsibles e incomodidades derivadas del experimento, los costos, las fuentes de financiación de la investigación, objetivos, métodos, posibles conflictos de intereses, afiliaciones institucionales del investigador, y todo otro aspecto pertinente de la investigación o aquella que de manera particular requiera el individuo, con verificación de que ésta ha sido entendida $)^{55,56,57}$ este proceso finaliza con la solicitud de consentimiento por parte del profesional o persona calificada y con la decisión libre, informada, previa y expresa, de participar o no en el proceso de investigación, la cual idealmente debe ser por escrito. ${ }^{" 58,59}$ La información se extiende al finalizar los procesos de investigación, debido a que las personas tienen derecho a acceder a los resultados de ésta y a los beneficios generados. ${ }^{60}$ En el caso de informaciones con respecto al estado de la salud, se protege el derecho a no ser informado (extensivo a la familia afectada), con excepciones que propendan por el mejor interés del paciente. ${ }^{61}$ El derecho a la información se acompaña del deber de prudencia el cual se manifiesta cuando este proceso puede afectar de manera sustancial la salud del individuo, materializado en la necesidad de asesoría o consejería genética (de carácter no directivo, acorde con la cultura y con el interés superior del interesado). ${ }^{62}$

La autonomía tiene que ver también con el derecho que tiene todo individuo de acceder a su información genética y a que se respete su intimidad y vida privada en lo relativo a informaciones con respecto a su salud, es decir, que su información no sea revelada a terceros sin su autorización expresa (se hace especial énfasis en el caso de "aseguradores, empleadores, establecimientos de educación y familiares"), salvo cuando prime un interés mayor como el de la salud pública. En el caso de la información genética esta tiene un carácter confidencial y privado, por lo tanto debe garantizarse la seguridad en su manejo, esto incluye que por regla general, a no ser que sea estrictamente necesario para la investigación, los datos se encuentren disociados de la identificación de las personas en su almacenamiento, y que éste se haga únicamente por el tiempo estrictamente necesario, momento en el cual deben ser destruidos (especialmente con relación a procesos penales, civiles o de medicina forense). ${ }^{63,64}$ Así mismo, en el caso de contarse con resultados derivados de éstas, las personas tienen el derecho a ser o no informadas, lo cual debe ser aclarado antes que inicie la investigación (excepto en el caso

55 Declaración Internacional sobre Datos Genéticos Humanos. Art. $6^{\circ}$.

56 Declaración de Helsinki. Art. 24, 34.

57 Código de Nüremberg. Art. $1^{\circ}$.

58 Declaración Internacional sobre Datos Genéticos Humanos. Art. $8^{\circ}$.

59 Declaración de Helsinki. Art. 24.

60 Declaración de Helsinki. Art. 33.

61 Declaración Internacional sobre Datos Genéticos Humanos. Art. 10.

62 Declaración Internacional sobre Datos Genéticos Humanos. Art. 11.

63 Declaración Internacional sobre Datos Genéticos Humanos. Art. 14.

64 Declaración Internacional sobre Datos Genéticos Humanos. Art. 21. 
de datos irreversiblemente disociados de personas identificables o que no permitan sacar conclusiones particulares) ${ }^{65}$.

Los seres humanos son propietarios de su información genética, tienen el derecho a que se proteja el acceso a éstos, más aún cuando median fines comerciales. De otra parte, los desarrollos generados de las investigaciones tienen que estar protegidos por los derechos de propiedad intelectual incluidosel valor del conocimiento ancestral, el cual se fundamenta en la necesidad de solicitar el consentimiento de los "propietarios consuetudinarios o tradicionales de ese saber" ${ }^{66}$. El derecho a la información incluye todo aquello que represente un riesgo para la salud de las personas, como es el caso de los alimentos genéticamente modificados ${ }^{67,68}$.

c) Beneficencia, no maleficencia: el principio de beneficencia consiste en hacer el bien a las personas, balanceando en las acciones los riesgos con los beneficios, siempre desde la perspectiva de lo que represente el mejor interés desde la autonomía del sujeto, además, implica acción; el principio de no maleficencia consiste en no hacer daño, no provocar dolor o sufrimiento, no producir incapacidad y prevenir las situaciones de daño.

Estos dos principios no aparecen de manera expresa en las declaraciones y convenios pero, se encuentran de manera tácita expresados de distintas formas, algunas de estas son:

- El saber de la ciencia deber ser usado responsablemente, buscando satisfacer las "necesidades y aspiraciones del ser humano", el bienestar social, en el marco del desarrollo económico sostenible y la protección de la naturaleza, balanceando los riesgos con los beneficios en las dimensiones individuales, sociales y ambientales. La ciencia no debe tener un carácter caprichoso o innecesario ${ }^{69,70,71,72}$.

- Respeto y preservación por parte de la ciencia y su conocimiento de todas las formas de $v i d a^{73}$. Primacía de la dignidad, la libertad, el interés y el bienestar de la persona sobre los de la sociedad o la ciencia ${ }^{74,75,76,77}$.

65 Declaración Internacional sobre Datos Genéticos Humanos. Art. 10.

66 Declaración sobre la Ciencia y el uso del Saber Científico. Art. 38.

67 Declaración sobre la Ciencia y el uso del Saber Científico. Art. $8^{\circ}$.

68 Declaración Bioética de Gijón.

69 Declaración sobre la Ciencia y el uso del Saber Científico. Preámbulo 1. 11.

70 Declaración Bioética de Gijón.

71 Declaración sobre la Ciencia y el uso del Saber Científico. Art. 28.

72 Código de Nüremberg. Art. $2^{\circ}$.

73 Declaración sobre la Ciencia y el uso del Saber Científico. Art. 23.

74 Declaración Universal del Genoma Humano y los Derechos Humanos. Art. 10.

75 Declaración de Helsinki. Art. $6^{\circ}$.

76 Código de Nüremberg.

77 Declaración de Helsinki. Art. $3^{\circ}$. 
- Se debe evitar todo daño incapacitante grave, sufrimiento físico o psicológico, o muerte ${ }^{78}$.

- Definición de los límites específicos de la investigación en seres humanos, incluida la investigación en genética humana y la aplicación de técnicas de reproducción asistida (alivio del sufrimiento, mejora de la salud del individuo y la humanidad, comprensión de las causas, evolución y efectos de las enfermedades, mejora de las intervenciones preventivas, diagnósticas y terapéuticas, intervención sobre la esterilidad humana, diagnóstico y tratamiento de enfermedades de origen hereditario) ${ }^{79,80,81}$.

- Límites a la modificación del genoma humano (razones estrictamente de carácter preventivo, diagnóstico o terapéutico, siempre y cuando no se modifique el genoma de la descendencia o se usen las técnicas de reproducción asistida para la selección del sexo).

- Desarrollo de las actividades por profesionales con probada idoneidad, acorde con los principios científicos aceptados, las normas nacionales e internacionales, los estándares éticos, legales, jurídicos y profesionales y a la evidencia científica. Esto incluye en el caso de datos genéticos, proteómicos y muestras biológicas humanas la necesidad de garantizar la "exactitud, fiabilidad, calidad y seguridad" de estos y la responsabilidad del investigador de lo ocurrido en el proceso de investigación con respecto a las consecuencias éticas, jurídicas y sociales ${ }^{82,83,84,85,86,87,88,89}$.

- Formación y responsabilidad ética de los investigadores ${ }^{90,91}$ y desarrollo de la regulación deontológica para las profesiones que tienen que ver con la investigación en ciencias de la salud ${ }^{92}$.

- Establecimiento de condiciones específicas para la experimentación con seres humanos, que incluye: la no existencia de un método alternativo al experimento con una eficacia comparable, basada en experimentación en animales y en la historia natural de la enfermedad; riesgos que no sean desproporcionados con los beneficios potenciales; aprobación del proyecto por autoridad competente; previo estudio independiente sobre

\footnotetext{
78 Código de Nüremberg. Arts. $4^{\circ}, 5^{\circ}$.

79 Declaración Universal del Genoma Humano y los Derechos Humanos. Art. 12.

80 Declaración de Helsinki. Art. $7^{\circ}$.

81 Declaración de Gijón.

82 Declaración Universal sobre el Genoma Humano y los Derechos Humanos. Art. $5^{\circ}$.

83 Declaración Internacional sobre los Datos Genéticos Humanos. Art. 15.

84 Declaración de Helsinki. Art. $9^{\circ}$.

85 Declaración de Helsinki. Arts. 10, 12.

86 Declaración de Helsinki. Art. 16.

87 Código de Nüremberg. Art. $8^{\circ}$.

88 Código de Nüremberg. Art. $7^{\circ}$.

89 Declaración de Derechos Humanos. Art. $3^{\circ}$.

90 Declaración Internacional sobre los Datos Genéticos Humanos. Art. 24.

91 Declaración sobre la Ciencia y el uso del Saber Científico. Art. 21.

92 Declaración sobre la Ciencia y el uso del Saber Científico. Art. 41.
} 
su pertinencia científica (importancia del objetivo del experimento y el estudio multidisciplinar de su aceptabilidad en el plano ético); información sobre derechos y garantías a quienes participen en la investigación; consentimiento libre, explícito, por escrito y que pueda ser retirado en cualquier momento ${ }^{93}$.

- Evaluación de los riesgos, costos y beneficios previo al inicio de la investigación (balance riesgo-costo-beneficio), evaluación si los riesgos pueden ser fácilmente superados antes de iniciar la investigación; beneficios directos en el bienestar de las personas que participan en la investigación; suspensión en caso de que los riesgos sean mayores que los beneficios o con la existencia de resultados concluyentes; "El grado de riesgo que se corre nunca podrá exceder el determinado por la importancia humanitaria del problema que el experimento pretende resolver." $94,95,96$

- Desarrollo de la investigación en seres humanos en el marco de protocolos de investigación, en el que se incluye información que pueda evidenciar conflictos de interés ${ }^{97}$.

- Aprobación y seguimiento por parte de comités de ética multidisciplinarios independientes, plurales y transparentes, que obren en el marco de la regulación nacional vigente así como de la internacional (incluso se prevé la figura de interacción de comités de distintos Estados cuando se trate de investigación multicéntrica). Se crea así mismo el Comité Internacional de Bioética (CIB) y el Comité Intergubernamental de Bioética (CIGB) ${ }^{98,99,100,101}$.

- Respeto a la integridad y vulnerabilidad humana. La participación de poblaciones vulnerables sólo se justifica cuando éstas se vean beneficiadas directamente ("éstas incluyen a los que no pueden otorgar o rechazar el consentimiento por sí mismos y a los que pueden ser vulnerables a coerción o influencia indebida") ${ }^{102,103}$.

- Mecanismos de supervisión y gestión de los datos genéticos ${ }^{104}$.

- Reparación equitativa por los daños injustificados producidos ${ }^{105}$.

d) Justicia: la mayoría de declaraciones utilizan la palabra acceso equitativo, igualitario o justicia, para referirse a la necesidad de que la humanidad

93 Código de Nüremberg. Art. $3^{\circ}$.

94 Declaración de Bioética y Derechos Humanos. Art. $4^{\circ}$.

95 Declaración de Helsinki. Arts. 18, 20, 21.

96 Código de Nüremberg. Arts. 6, 10.

97 Declaración de Helsinki. Art. 14.

98 Declaración Universal del Genoma Humano y los Derechos Humanos. Art. 16.

99 Declaración de Bioética y Derechos Humanos. Art. 19.

100 Declaración Internacional sobre los Datos Genéticos Humanos. Art. $6^{\circ}$.

101 Declaración de Helsinki. Art. 15.

102 Declaración de Bioética y Derechos Humanos. Art. $8^{\circ}$.

103 Declaración de Helsinki. Art. $9^{\circ}$.

104 Declaración Internacional sobre los Datos Genéticos Humanos. Arts. 20, 25.

105 Declaración Universal del Genoma Humano y los Derechos Humanos. Art. $8^{\circ}$. 
en su conjunto puede beneficiarse de los productos y servicios generados de la investigación en genética humana, independiente de sus costos y del carácter privado de su desarrollo ${ }^{106}$. Otra manera de garantizar la justicia, como se describió anteriormente en el análisis de los principios de beneficencia y no maleficencia, consiste en que la población que participa en los estudios de investigación pueda gozar de beneficios directos de los resultados o aplicaciones obtenidas, y no sólo de los riesgos; esta premisa es aplicable también al uso de la información genética producto de la biodiversidad aún conservada (a expensas de un relativo "atraso en el desarrollo económico") de los países en vías de desarrollo.

El principio de justicia también expresa la garantía de participación en los estudios de investigación de las personas subrepresentadas ${ }^{107}$.

Llama la atención que otros principios tengan un desarrollo preponderante a lo largo de las declaraciones y convenios, y la justicia, a pesar de ser mencionada no sea tan evidente; por esto, en el marco del derecho internacional propio de los procesos de globalización, algunos autores mencionen que se han "globalizado los beneficios y el comercio pero no la justicia."108

e) Respeto a la diversidad: se aplica a sus distintas manifestaciones (biológica, genética, cultural), es un principio presente en la mayoría de las declaraciones, se le reconoce como necesaria y parte del patrimonio de la humanidad ${ }^{109,110,111,112}$. El Convenio sobre la Diversidad Biológica menciona el término "recurso genético" como elemento relacionado con la biodiversidad y establece lineamiento para su uso ${ }^{113}$. Ésta, expresada como pluralismo, se reconoce en la constitución de los comités de ético-regionales, nacionales e institucionales, debido a que los dilemas generados por la interacción de la vida con la tecnología están profundamente marcados en su resolución en un sentido u otro por las posiciones ideológicas, incluyendo las religiosas. La pluralidad es a su vez un antídoto contra otras formas de discriminación y de fundamentalismo científico, contra la creencia que la verdad está sólo en manos de unos pocos.

Los riesgos en la aplicación de este principio son el de caer en el relativismo o en el particularismo; como menciona Romeo CASABONA en Hacia un derecho transcultural para la genética y la biotecnología humanas son múltiples las dificultades para encontrar principios que fundamenten una ética universal

106 Declaración Universal del Genoma Humano y los Derechos Humanos. Art. 12.

107 Declaración de Helsinki. Art. $5^{\circ}$.

108 Carlos M. Romeo Casabona Hacia un derecho transcultural para la genética y la biotecnología humanas. Anuario Jurídico de la Rioja. Número 12 del 2007. Págs. 11-34, cita a Sulston. Society and human genoma. Law and the Human Genome Review. Número 20. (2004). 32.

109 Declaración de Derechos humanos. Arts. 12, 17.

110 Declaración Universal sobre el Genoma Humano y los Derechos Humanos. Art. $1^{\circ}$.

111 Declaración sobre la Ciencia y el uso del Saber Científico. Preámbulo. Art. 35.

112 Declaración de Bioética y Derechos Humanos. Arts. 12, 17.

113 Convenio sobre la Diversidad Biológica. Junio de 1992. 
incluso en aquellos invocados para este fin como el de la reciprocidad, exhortado por Peter Singer, en One World, Ethics for Globalitation, siendo los derechos humanos y en el caso concreto de la biomedicina las declaraciones sobre el genoma humano y los datos genéticos aquellas que podrían cumplir con este papel unificador con la debilidad que expresan una visión occidental individualista, centrada en la persona y no en la colectividad.

f) Cooperación, solidaridad, equidad: estos principios tienen como principal objetivo vencer las inequidades en la producción y acceso a los beneficios generados de la investigación en genética humana entre naciones desarrolladas y en vías de desarrollo (la ciencia "debe convertirse en un bien compartido solidariamente" entre todas la naciones) ${ }^{114,115}$, la protección contra posibles abusos y la particularización del conocimiento a las necesidades específicas, así como la protección de poblaciones con especial vulnerabilidad ocasionada por enfermedades de índole genética, expresado en acuerdos bilaterales y multilaterales, en las dimensiones "intergubernamental, gubernamental y no gubernamental" 116 . De igual manera, se promueve el libre intercambio de conocimiento (que incluye incentivos a la publicación), la evaluación de riesgos y de beneficios, aprovechamiento compartido de los beneficios de la investigación por parte de los sujetos de investigación y la sociedad $d^{117,118,119,120}$.

g) Protección a las generaciones futuras: el genoma humano es considerado patrimonio de la humanidad, sin que en la Declaración de Genoma Humano y Derechos Humanos y en la Declaración Internacional de Datos Genéticos Humanos sean mencionadas expresamente las generaciones futuras.

La declaración de bioética y derechos humanos menciona expresamente las generaciones futuras, al referirse al impacto de la ciencia de la vida en la "constitución genética" de las futuras generaciones ${ }^{121}$. La declaración sobre la ciencia y el uso del saber científico también hace mención de la responsabilidad con las futuras generaciones ${ }^{122}$.

La declaración sobre responsabilidades de las generaciones actuales para con las generaciones futuras establece la responsabilidad que las generaciones presentes tienen con las futuras con respecto a garantizar la "salvaguardia de las necesidades y los intereses de las generaciones presentes y futuras", la libertad de elección, "el mantenimiento y la perpetuación de la humanidad",

\footnotetext{
114 Declaración sobre la Ciencia y el uso del Saber Científico. Arts. 8, 14, 35.

115 Declaración de Bioética de Gijón.

116 Declaración sobre la Ciencia y el uso del Saber Científico. Art. 36.

117 Declaración Universal del Genoma Humano y los Derechos Humanos. Arts. 17, 19.

118 Declaración de Bioética y Derecho Humanos. Art. 15.

119 Declaración Internacional sobre los Datos Genéticos Humanos. Arts. 18, 19.

120 Declaración Internacional sobre los Datos Genéticos Humanos. Art. 23.

121 Declaración de Bioética y Derechos Humanos. Art. 16.

122 Declaración sobre la Ciencia y el uso del Saber Científico. Preámbulo 1.
} 
y los recursos necesarios para la sobrevida, con mención especial del genoma humano y diversidad biológica. ${ }^{123}$

h) Precaución: un hallazgo llamativo es la ausencia del principio de precaución en las más importantes declaraciones y convenios relacionados con la investigación en genética humana (las declaraciones Internacional de Derechos Humanos, de Genoma Humano y Derechos Humanos, Internacional de Datos Genéticos Humanos, sobre Bioética y Derechos Humanos), que contrasta con su mención en las declaraciones, convenios y tratados internacionales relacionados con la protección del medio ambiente y la bioseguridad ${ }^{124}$.

En algunas de estas declaraciones o convenios sobre el genoma humano se habla de la seguridad implícita a este tipo de procesos que intervienen sobre la materia viva, asociada frecuentemente con el principio de precaución. La Declaración sobre la Ciencia y el uso del Saber Científico advierte de los riesgos de la interacción de la vida con la tecnología, los cuales incluso podrían poner en peligro la misma supervivencia del hombre, sin llegar a utilizar el principio de precaución dentro del texto ${ }^{125}$. En la Declaración de Bioética de Gijón se habla expresamente de la precaución como principio inspirador de las biotecnologías (con referencia específica a los alimentos genéticamente modificados que pudiesen afectar la salud humana) ${ }^{126}$.

Una de las más importantes dimensiones del riesgo en las aplicaciones biotecnológicas es la incertidumbre, caracterizada por el conocimiento incompleto, la ignorancia e indeterminación (debido al origen multicausal o al comportamiento caótico de las causas) ${ }^{127}$. El filósofo alemán HANS JonAs plantea que ante la precariedad y vulnerabilidad de la vida y el daño que el dominio del hombre ha producido en la naturaleza, éste debe soportar la carga de la libertad, la responsabilidad sobre sus actos ${ }^{128,129}$. JonAs recurre entonces a la heurística del miedo, la cual expresa el temor de la combinación de un poder técnico desmesurado, un vacío ético y un mínimo conocimiento, la cual implica "consultar antes a nuestros temores que a nuestros deseos". La ética de la responsabilidad implica, paralelo al desarrollo del saber técnico científico, un saber predictivo que permita predecir el riesgo en términos de gravedad y probabilidad, en escenarios futuros que evidencian las conse-

123 Protocolo de Cartagena sobre Seguridad de la Biotecnología del Convenio sobre la Diversidad Biológica http://www.cbd.int/doc/legal/cartagena-protocol-es.pdf

124 Declaración sobre la Ciencia y el uso del Saber Científico. Art. 20.

125 Declaración de Bioética de Gijón.

126 Andy StirLing. Science, precaution, and practice. Public Health Rep. 117:521-33. (2002).

127 Hans Jonas. The phenomenon of life: toward a philosophical biology. New York. Harper and Row. (1966).

128 Hans Jonas. El principio de responsabilidad: ensayo de una ética para la civilización tecnológica. Barcelona. Herder. (1995).

129 Informe Brundtland Nuestro Futuro Común Comisión Mundial para el Medio Ambiente y Desarrollo Naciones Unidas 1987. 
cuencias de las acciones. Por esto, si existen dudas con respecto a los efectos futuros, debe prevalecer la heurística del temor: en caso de duda se obra acorde con el escenario más catastrófico. El problema radica en la complejidad del mundo vital, el cual no se rige bajo las normas de causa-efecto.

Combinar cosas preciosas (personas) con peligrosas, tarde o temprano ocasiona problemas; del conocimiento incierto y de la relación que necesariamente existe entre hombre y naturaleza, y que no está exenta de riesgo (ninguna actividad humana tiene riesgo cero) e incluso traspasa el espacio temporal y se proyecta en el futuro como una amenaza a la misma existencia del hombre, aparece otro principio fundamental en la regulación de la aplicación de los desarrollos biotecnológicos, el de la precaución o de la cautela, que sirve de engranaje entre la ciencia y la técnica, entre el conocimiento y la acción. La precaución o cautela se aplica específicamente en el ámbito de la incertidumbre ante la posibilidad de causar daños graves e irreversibles (relacionados con el carácter no renovable del recurso, a largo plazo o de alto costo o cuando exista el riesgo de que pequeños perjuicios acumulados produzcan a largo plazo un riesgo irreversible) en lo referente a la salud, medio ambiente, bioseguridad y seguridad alimentaria, en el marco de la incertidumbre científica; parte de la posibilidad de que se cometan errores.

El principio de precaución se acompaña de la libertad de pensamiento y la libertad en la investigación, los cuales propenden por el desarrollo científico, relacionado con el desarrollo sostenible (que satisface las necesidades del presente sin comprometer la capacidad de las generaciones futuras de satisfacer las suyas) ${ }^{130}$, que beneficia a la humanidad.

i) Libertad científica: se reconocen los inmensos beneficios que la investigación en genética humana aporta a la calidad y esperanza de vida de las personas, así como la necesidad de preservar la libertad científica, dándosele un cariz neutro, es decir, una cosa es el conocimiento (ciencia) y otra su aplicación (técnica); la dificultad radica en que precisamente en las ciencias biotecnológicas, como es el caso de la genómica, existe un límite muy difícil de establecer, casi una fusión entre el conocimiento y su aplicación, de ahí la necesidad de establecer unos límites éticos, que la promuevan pero que la cuestionen y direccionen permanentemente con respecto a su servicio en pro del bienestar de las personas y a la paz, la salvaguarda de la dignidad y los derechos humanos y su desarrollo dentro de los más altos estándares científicos. Esto supone una serie de responsabilidades especiales para los investigadores.

JuAn RAmón LACAdEna, citando a SteTten, compara la libertad de investigación con la libertad de expresión, reconociendo su calidad de derecho

130 Declaración Universal del Genoma Humano y los Derechos Humanos. Arts. 12, 13, 14, 15. 
inalienable con límites: “... es una cosa preciosa digna de ser alimentada y cuidada, pero, como otras libertades, no es absoluta. Todos sabemos que la libertad de expresión puede ser coartada cuando su ejercicio puede engendrar un peligro real; "todos hemos sido educados en la norma de que no se puede gritar 'fuego' en un teatro abarrotado de público"...".

j) Respeto a la propiedad intelectual: paralelo a la protección de la libertad científica se encuentra el respeto a la propiedad intelectual, producto de los resultados de investigación en genética humana, en un sano equilibrio con el acceso a la información por parte de países en desarrollo; la protección incluye los conocimientos ancestrales ${ }^{131}$.

k) Inclusión y participación: aunque como los principios de inclusión y participación no aparecen en las declaraciones, hemos dado esta denominación a una guía específica que se traduce de sus lecturas, en razón de que se insta a que las personas en su conjunto tengan acceso a la información, la educación y la formación referentes al tema, así como a los aspectos éticos y bioéticos, como una forma de hacer vivos los principios enunciados y de favorecer la participación de los ciudadanos a través de debates plurales e inclusivos, debido al impacto en las distintas dimensiones de la vida humana que tiene la investigación en la genética humana y a la rapidez con la que se producen y se aplican los desarrollos biotecnológicos, en los cuales deben participar científicos, políticos, ciudadanos, medios de comunicación, las distintas disciplinas y los sectores privado y público, que permitan enfrentar los distintos dilemas éticos asociados, y que orienten la investigación pertinente ${ }^{132,133,134}$. De otra parte, existe una obligación ética de publicar los resultados de investigación en seres humanos (positivos, negativos e inconclusos, así como la información que pueda revelar posibles fuentes de conflicto de interés) ${ }^{135}$.

131 Declaración sobre la Ciencia y el uso del Saber Científico. Art. 38.

132 Declaración Universal sobre el Genoma Humano y los Derechos Humanos. Arts. 20, $21,23,24$.

133 Declaración sobre la Ciencia y el uso del Saber Científico. Arts. 4º 22.

134 Declaración de Gijón.

135 Declaración de Helsinki. Art. 30. 


\section{CONCLUSIONES}

Las intervenciones biotecnológicas sobre el genoma humano están asociadas a la posibilidad de materialización de riesgos graves e irreversibles, con efectos globales y transgeneracionales (que incluso pueden afectar bienes inexistentes como el patrimonio genómico de futuras generaciones) y que pueden ser modificados en el tiempo gracias a los constantes avances tecnológicos.

La respuesta del derecho a las intervenciones biotecnológicas sobre el genoma humano es diversa e incluye prohibir, permitir u obligar, pero dado el carácter emergente y en permanente cambio de los hechos derivados de la biotecnología, podrían presentarse situaciones que dejen en evidencia lagunas en la normativa jurídica; los jueces podrían enfrentar situaciones en las cuales el derecho positivo no sea suficiente para resolver un conflicto de manera eficaz y segura. En estos casos bien podrían aplicarse las normas existentes (autointegración), o rechazar la regulación y resolver las situaciones jurídicas por la vía de la heterointegración ${ }^{136}$. Para CARlos María Romeo Casabona, en este contexto, el derecho debe "asumir las relaciones sociales emergentes, positivizando, regulando, o reconociendo (judicialmente) dichas relaciones; o bien aplicando a la nueva situación, principios generales básicos ya integrados jurídicamente, como son los de respeto a la autonomía individual y a la dignidad de la persona, o incorporando nuevos principios axiológicos". En este orden de ideas, los principios jurídicos, dado su carácter más abierto, podrían ser de mayor utilidad.

El derecho internacional relacionado con las intervenciones sobre el genoma humano desarrollado por diversos organismos como la Organización de Naciones Unidas o la Unesco, se expresa a través del derecho declaratorio o soft law o derecho de referencia (opuesto al derecho instrumento que fundamentado en leyes, decretos, directivas que juridifican la sociedad), el cual, si bien contempla lineamientos con respecto a las intervenciones sobre el genoma humano, no tiene en una primera aproximación un carácter obligatorio y depende de la voluntad de los países para su acatamiento, bien sea a través de su incorporación a las regulaciones internas o servir a través de un carácter analógico, como guía en la resolución de situaciones de frontera ${ }^{137}$.

Las declaraciones procuran expresar los principios éticos, es decir, las ideas fundamentales que en lo moral orienten a los Estados, personas, grupos e instituciones en la toma de decisiones, de tal manera que estas propendan por el bien del hombre como sujeto individual y como parte de la humanidad,

136 Miguel Ángel Ciuro. Cuestiones axiológicas críticas en el desarrollo del bioderecho. Revista Bioética y Derecho. Primeras Jornadas Argentinas de Bioética-Primeras jornadas Latinoamericanas de bioética. Pág. 23.

137 Noelle Lenoir. El genoma humano, la ética y los derechos humanos. Pág. 131. Diálogo México. Número 23. (1998). 
presente y futura (hecho que de por sí genera una complejidad en el desarrollo ético y jurídico) y que de alguna forma expresan un consenso mínimo. Los principios jurídicos buscan proteger el ser humano en lo referente a su dignidad, identidad, integridad (personal y colectiva), individualidad, igualdad, intimidad, equidad y autonomía en relación con los adelantos científicos; procuran balancear la protección de la dignidad humana con la libertad científica coherente con el progreso social, de tal forma que se equilibren los riesgos con los beneficios, en un marco de seguridad determinado por el principio de precaución ${ }^{138}$.

En este sentido, el derecho genético debe acoger estos principios para proteger derechos de primera generación (civil y política), segunda generación (social y económica), tercera generación (paz y desarrollo ambiental) y cuarta generación (derechos intergeneracionales). Algunos de los derechos que deben ser protegidos son: a la identidad genética, a la integridad genética personal y de otros sistemas vivos así como futuras generaciones, a la intimidad, a la imperfección o al no perfeccionamiento (se asocia al derecho a nacer de forma natural), a la diversidad genética, (clave de la supervivencia de la vida), a la variabilidad humana (visión contraria al determinismo genético), al acceso equitativo a los recursos biotecnológicos, a la imparcialidad en la toma de decisiones que implican la información genética personal, a ser informado o no con respecto al riesgo genético a través de proceso de consejería genética, a no ser discriminado por razones genéticas, a un desarrollo sostenible, a la unicidad, igualdad y comunidad de los seres humanos, entre otros.

Entendiendo las diferencias existentes entre los principios éticos y jurídicos, cabe preguntarse si los principios éticos que orientan el diseño de las declaraciones sobre el genoma humano coinciden, en el ámbito del derecho genético referido a las intervenciones sobre éste, con los principios jurídicos generales o si algunos de los principios enunciados son optativos, dado su carácter ético. El problema se hace más complejo cuando estos principios son integrados o interpretados a la luz de las decisiones jurídicas nacionales, debido a las múltiples miradas que coinciden en un mundo globalizado, las cuales incluso son cambiantes en el tiempo dentro de una misma sociedad debido, entre otros, a los mismos efectos de la biotecnología, que hace que los principios enunciados en relación con la intervención sobre el genoma humano no sean inmutables y tengan un carácter cambiante o progresivo.

Así como se ha tratado de establecer la importancia de contar con una serie de principios jurídicos en relación con el derecho genético, también se debe aclarar que frente a una serie de riesgos graves e irreversibles, hay que

138 Gonzalo Figueroa Yánez. Hacia una integración supranacional de los principios rectores sobre el genoma humano. Una visión personal desde la perspectiva latinoamericana. Págs. 231 a 245. Acta Bioethica. Año VI. no 2. (2000). 
contar con reglas jurídicas que eviten la vulneración de los derechos individuales y colectivos.

El análisis del marco declaratorio internacional deja evidenciar elementos que permiten la caracterización del derecho genético en lo relacionado con las intervenciones sobre el genoma humano:139

La existencia de sujeto activo (cualquier persona técnica o profesional que con conocimientos médicos realiza intervenciones genéticas en otros sujetos humanos), de un sujeto pasivo (cualquier persona en la que recae la acción y de manera excepcional lo pueden ser células, elementos celulares, germinales, cigotos, preembriones, embriones, fetos, tejidos, órganos o cadáveres) y objeto (regula la aplicación de procedimientos genéticos en relación con el ser humano, permitiendo una orientación humanística de las técnicas biotecnológicas, con una dimensión individual y colectiva, que incluye a futuras generaciones).

\section{Protección a bienes jurídicos como la vida, la intimidad, la libertad, la autonomía}

La dimensión del impacto de las intervenciones sobre el genoma en las distintas etapas de la vida humana (económicas, políticas, sociales, ideológicas, entre otras), implica la constante relación del derecho genético con otras áreas del derecho como la civil, penal, constitucional, de seguros, informáticos, infancia, familia, industrial, laboral, mercantil, ambiental, administrativos, derechos humanos, constitucional e internacional, entre otros.

A diferencia de otras áreas de las ciencias jurídicas, el derecho genético debe regular hechos que aún no han ocurrido, sobre los cuales inclusive la ciencia no ha perfeccionado los medios para que se materialicen. Por esto debe extender su horizonte hacia situaciones futuras y proteger bienes inexistentes (patrimonio genómico de las futuras generaciones). De alguna manera se relaciona con el análisis temporal del derecho y con la reconstrucción histórica que de éste se hace, pero en una línea temporal opuesta que se alimenta de la prospectiva. La dinámica del cambio del derecho es, sin embargo, una tarea en construcción de los teóricos del derecho ${ }^{140}$.

Dado que los riesgos relacionados con este tipo de intervenciones afectan a la humanidad en su totalidad, el desarrollo regulatorio debe estar fundamentado en un amplio debate social, en el cual la bioética dentro de los límites de la disciplina juega un papel fundamental. Su expresión se hace en

139 Enrique Varsi Rospigliosi. Fundamentos básicos del derecho genético. issuu.com/evarsi/docs/varsicap $2 / 5-18$

140 Hernando Gutiérrez Prieto. Equilibrio jurídico, bases para la conceptualización en una teoría dinámica del sistema jurídico. Universitas. Número 116 del 2008. Pág. 254. 
su mayoría a través de declaraciones internacionales, por lo que se requiere dar valor jurídico del marco declaratorio internacional tipo soft law.

Es altamente biotecnificado y se mueve en la frontera del conocimiento, podría decirse que se trata de un sistema de conocimiento abierto que se nutre de los permanentes desarrollos biotecnológicos, así como de las posturas valorativas de la sociedad.

$\mathrm{Al}$ igual que en otras áreas del derecho, los sistemas jurídicos resolverán estos conflictos basados en diferentes fuentes como la constitución, las leyes, la costumbre del lugar, las disposiciones que regulan casos semejantes o materias análogas y la regulación de carácter internacional.

Para finalizar, la revisión de los principios ético-jurídicos en las declaraciones internacionales permite deducir algunas de las áreas temáticas que requieren desarrollo regulatorio dentro de los sistemas jurídicos nacionales, los cuales deben hacer parte del derecho genético en lo referente a la intervención sobre el genoma humano y sus usos: ${ }^{141}$

- Investigación en ciencia y tecnología e investigación con seres humanos y comunidades.

- Intervención genética (la cual incluye la terapia génica, desarrollo de productos biotecnológicos, intervención de embriones, producción de quimeras e híbridos, clonación y eugenesia, medicina reproductiva y donación de gametos).

- Tratamiento y protección de la información genética (que incluye aspectos como el consentimiento informado para la obtención, conservación y circulación de la información, derecho a ser o no ser informado, manejo de biobancos de genes, material proteómico, células madre, embriones y material biológico humano como órganos y tejidos, protección contra la discriminación fundada en razones genéticas, uso con fines legales: pruebas de paternidad y de acciones criminales).

- Patentes sobre material genético y propiedad intelectual (patentes biotecnológicas).

- Protección a la diversidad y aspectos de bioseguridad.

- Creación de instituciones encargadas del diseño de políticas, asesoría, evaluación y control.

- Control sobre la idoneidad profesional de quienes intervienen sobre el genoma humano. 


\section{BIBLIOGRAFÍA}

Mercedes Alberruche DíAz-Flores. La clonación y selección de sexo ¿? Ed. Dykinson. Madrid. España. (1998).

Ángela Aparisi Miralles. Bioética, bioderecho y biojurídica, reflexiones desde la filosofía del derecho. Pág. 63. Anuario de filosofía del derecho. ISSN 0518-0872, nº 24. (2007).

JoAquín Arce y FlóRez-VAldés. Los principios generales del derecho y su formulación constitucional. Pág. 99. Madrid. Editorial Civitas, S.A.

Tom Beauchamp \& James Chidress. Principles of Biomedical Ethics. Nueva York. Oxford University Press. (1979).

Luisa Benavides de Castañeda. Bioética y bioderecho: temas para la reflexión. Pág. 91. Anuario $\mathrm{n}^{\mathrm{o}}$ 30. ISSN 1316-5852. (2007).

Darío Bergel Salvador. Genoma humano. www.bibliojuridica.org/libros/1/211/7.pdf

A. CAmbrón. Génesis y problemas de la bioética. En A. CAmbrón (coord.). Entre el nacer y el morir. Págs. 9 y ss. Comares. Granada. (1998).

J. M. Serrano Ruiz-Calderón. Bioética y derecho. En Gloria Tomás Garrido. Manual de Bioética. Op. cit. Pág. 60.

Miguel Ángel Ciuro. Cuestiones axiológicas críticas en el desarrollo del bioderecho. Revista Bioética y Derecho. Primeras Jornadas Argentinas de Bioética-Primeras jornadas Latinoamericanas de bioética. Pág. 23.

Comisión Mundial para el Medio Ambiente y Desarrollo Naciones Unidas. Informe Brundtland Nuestro Futuro Común. (1987).

Francesco D'Agostino. "La bioética como problema jurídico”, Bioética. Estudios de filosofía del derecho. Op. cit. Pág. 76.

GonZalo Figueroa YÁnez. Hacia una integración supranacional de los principios rectores sobre el genoma humano. Una visión personal desde la perspectiva latinoamericana. Págs. 231 a 245. Acta Bioethica. Año VI. no 2. (2000).

Diego Gracia Guillen. Fundamentos de bioética. Pág. 576. Eudema. Madrid. (1989).

Hernando Gutiérrez Prieto. Equilibrio jurídico, bases para la conceptualización en una teoría dinámica del sistema jurídico. Universitas. Número 116 del 2008. Pág. 254.

Informe Brundtland. Nuestro futuro común. Comisión Mundial para el Medio Ambiente y Desarrollo Naciones Unidas (1987).

Héctor Gros Espiell. Ética, bioética y derecho: ética y derecho. Pág. 87. Editorial. Temis. (2005).

Roberto Marino Jiménez Cano. Sobre los principios generales del derecho. Especial consideración en derecho español. Revista Telemática de Filosofía del Derecho. no 3. 1999/2000. Pág. 1. D.L. M-32727-1998.

Joan Mead Stephanie. The Precautionary Principle: A Discussion of the Principle's Meaning and Status in an Attempt to Further Define and Understand the Principle. New Zealand Journal of Environmental Law. (2004). Pág. 137. 
Hans Jonas. The phenomenon of life: toward a philosophical biology. New York. Harper and Row. (1966).

El principio de responsabilidad: ensayo de una ética para la civilización tecnológica. Herder. Barcelona. (1995).

Karl Larenz. Metodología de la ciencia del derecho. Pág. 418. Editorial Ariel, S.A. Barcelona. (1994). Traducción y revisión de Marcelino Rodríguez Molinero ( $1^{\mathrm{a}}$ ed. en Ariel, $3^{\mathrm{a}}$ ed. española).

Lenoir Noelle. El genoma humano, la ética y los derechos humanos. Diálogo México. Número 23. Abril de 1998. Pág. 131.

Fernando Lolas Stepke. Bioética, el diálogo moral en las ciencias de la vida. $2^{\mathrm{a}}$ Edición. Santiago de Chile. Mediterráneo Ltda. (2001).

Bioética. El diálogo moral en las ciencias de la vida. $2^{\mathrm{a}}$ Edición. Santiago de Chile. Mediterráneo Ltda. (2001).

Mariano Moreno Villa. Dignidad de las personas en el diccionario del pensamiento contemporáneo. Págs. 359-368.

Organización de las Naciones Unidas para la Educación, la Ciencia y la Cultura. La clonación humana cuestiones éticas. SHS-2006/WS/19. (2004). http://www.unesco.org/bioethics

Van Rensselaer Potter. "Bioethics. The science of survival”. Perspectives in Biology and Medicine. Págs. 127-153. New York. (1970).

. Bioethics: bridge to the future, Prentice Hall, Englewood Cliffs, Nueva Jersey. (1971).

Raffensperger Carolyn. Precaution and Security, the Labyrinthine Challenge. Pág. 34. Whole Earth (2002).

Eduardo Rodríguez Yunta. Desarrollo e innovación tecnológica, ética de fines y medios. En el libro Ética e innovación tecnológica. Editor Fernando Lolas S. Págs. 35-43. CIEB. Universidad de Chile. (2006). www.uchile.cl/bioetica/doc/libroeticaeinn.pdf

Ramón Martín Mateo. Bioética y derecho. Ariel. Barcelona. (1987).

Carlos M. Romeo Casabona. Hacia un derecho transcultural para la genética y la biotecnología humanas. Anuario Jurídico de la Rioja. Número 12 (2007), Págs. 11-34. Cita a Sulston, Society and Human Genoma. Law and the Human Genome Review. Número 20. (2004).

CARlos Ruiz Miguel. La nueva frontera del derecho a la intimidad: genética y dignidad. http:// web.usc.es/ ruizmi/pdf/genet.pdf

Chris SAnder. Genomic Medicine and the Future of Health Care. 287, 5460. Science. Mar 17. (2000).

Andrew Stirling. Science, Precaution, and Practice. Págs. 521-33. Public Health Rep. (2002).

Diego Valadés. Problemas del bioderecho y del derecho genómico. Panorama sobre la legislación en materia de genoma humano en América Latina y el Caribe. Pág. 389. (2006) http:// www.bibliojuridica.org/libros/5/2265/15.pdf

Enrique Varsi Rospigliosi. Fundamentos básicos del derecho genético. issuu.com/evarsi/docs/ varsi-cap $2 / 5-18 k$

Marciano Vidal. Bioética. Estudios de bioética racional. Pág. 16. Tecnos. Madrid. (1989). 
Rodolfo VảsQuez. Bioética y derecho: fundamentos y problemas actuales. Juridificar la bioética. Fondo de la Cultura Económica de México. (1999).

María Victoria Vidal CASERo. El proyecto genoma humano, sus ventajas, sus inconvenientes y sus problemas éticos. Cuadernos de bioética. Págs. 393. (Ejemplar dedicado a: ¿En qué fundamentamos la bioética?). (2001).

\section{Declaraciones y convenios}

Código de Nüremberg. Tribunal Internacional de Nüremberg. (1947).

Convenio sobre la Diversidad Biológica en la Conferencia de las Naciones Unidas sobre el Medio Ambiente y el Desarrollo, junio de 1992.

Declaración de Bioética y Derechos Humanos. Aprobada por la Conferencia General de la Unesco, octubre del 2005. Art. 11.

Declaración de Bioética de Gijón. Sociedad Internacional de Bioética. Comité Científico Congreso Mundial de Bioética (Gijón, España, 2000).

Declaración de Helsinki. Asociación Médica Mundial. Adoptada en 1964, último ajuste Asamblea General de octubre 2008.

Declaración Internacional sobre los Datos Genéticos Humanos. Unesco. Aprobado por la Conferencia general en París, 16 de octubre del 2003. Art. $1^{\circ}$.

Declaración sobre la Ciencia y el uso del Saber Científico. Unesco. Adoptada por la Conferencia Mundial sobre la Ciencia el 1 ${ }^{\circ}$ de julio de 1999.

Declaración sobre la clonación humana. Organización de Naciones Unidas, adoptada por la Asamblea General de las Naciones Unidas, el 23 de marzo del 2005.

Declaración Universal de Derechos Humanos. Organización de Naciones Unidas. Adoptada y proclamada por la Resolución de la Asamblea General 217 A (III) del 10 de diciembre de 1948.

Declaración Universal del Genoma Humano y los Derechos Humanos. Unesco. Aprobada el 11 de noviembre de 1997 por la Conferencia General en su 29a reunión.

Protocolo de Cartagena sobre Seguridad de la Biotecnología del Convenio sobre la Diversidad Biológica http://www.cbd.int/doc/legal/cartagena-protocol-es.pdf 